immunophenotype, response to prednisone, response to induction treatment, and presence of unfavorable genetic aberrations - to stratify 2,169 patients with ALL according to their risk of distant relapse and to risk-adjust their treatment strategies.

Despite receiving only half the dose of daunorubicin received by a historical control group from the ALL-BFM 90 trial, the standard-risk group (comprising 35\% of the study population) achieved a 6-year probability of event-free survival (pEFS) of $89.5 \%$, which is comparable with the pEFS of $88.7 \%$ achieved in the historical controls. In the medium-risk group (53\% of patients), the 6-year pEFS was $79.7 \%$. Notably, the omission of preventative cranial irradiation in medium-risk patients with non-T-cell ALL did not result in a significant reduction in 6-year pEFS (83.1\% in ALL-BFM 90, and 81.4\% in ALL-BFM 95). Unfortunately, treatment with cytarabine following consolidation failed to improve outcome in the 514 medium-risk patients randomized to receive this additional treatment. In the high-risk group (12\% of the population), the intensification of consolidation resulted in a 6-year pEFS of $53.2 \%$, a considerable improvement over that achieved in ALL-BFM 90 (31.9\%).

The authors conclude that in non-high-risk patients considerable reductions in treatment intensity can be achieved without adversely affecting outcome.

Original article Möricke A et al. (2008) Risk-adjusted therapy of acute lymphoblastic leukemia can decrease treatment burden and improve survival: treatment results of 2169 unselected pediatric and adolescent patients enrolled in the trial ALL-BFM 95. Blood 111: 4477-4489

\section{Simple molecular-grade gene index has prognostic value in early-stage breast cancer}

The prognostic value of histologic tumor grade is well established. Ma et al. have now reported a simple gene-expression index for tumor grade in breast cancer and have shown that this index in combination with the previously described HOXB13:IL17BR index provides more-accurate prognosis in early-stage breast cancer than achieved with either gene signature alone.

From a list of 39 genes with expression profiles that correlate with tumor grade and progression generated in a previous study, the researchers identified five genes that are involved in different phases of the cell cycle that were combined to form a molecular grade index. When used in two independent, publicly available microarray data sets generated from 410 patients with breast cancer, the five-gene index had similar prognostic capacity to the previously described 97 -gene genomic grade index. The researchers then developed a realtime reverse transcription polymerase chain reaction assay for the five-gene index and validated it in two further cohorts. This assay was able to distinguish grade I tumors from grade III tumors with an accuracy of $86 \%$; patients with grade III tumors benefited from chemotherapy, while those with grade I tumors did not. Among a cohort of lymph-node-negative patients treated with endocrine therapy, the prognostic power of the five-gene index was augmented when the HOXB13:IL17BR ratio was also considered; the combined index had the capacity to identify a unique subgroup of patients with a very high risk of experiencing distant metastasis.

The authors conclude that risk stratification of patients by use of the combined index identifies a subgroup of patients with early-stage breast cancer who would benefit from intense treatment regimens.

Original article Ma XJ et al. (2008) A five-gene molecular grade index and HOXB13:IL17BR are complementary prognostic factors in early stage breast cancer. Clin Cancer Res 14: 2601-2608

\section{Therapy with cetuximab and irinotecan in refractory metastatic colorectal cancer}

Agents that target the EGFR might be effective as second-line therapy in patients with metastatic colorectal cancer (mCRC) who are refractory to standard first-line treatments. In a multicenter, open-label, randomized phase III study, Sobrero et al. evaluated the effect of adding the EGFR antagonist cetuximab to irinotecan in patients with EGFR-expressing $\mathrm{mCRC}$ who were refractory to first-line fluoropyrimidine and oxaliplatin treatment but had not previously been treated with irinotecan. All participants received $300 \mathrm{mg} / \mathrm{m}^{2}$ or $350 \mathrm{mg} / \mathrm{m}^{2}$ irinotecan every 3 weeks, and half of the patients also received a single $400 \mathrm{mg} / \mathrm{m}^{2}$ dose of cetuximab followed by $250 \mathrm{mg} / \mathrm{m}^{2}$ per week. 